\title{
Pathology specimens over three decades
}

\author{
A survey of oral and maxillofacial pathology specimens submitted by general dental practitioners over a \\ 30-year period C. D. Franklin and A. V. Jones Br Dent J 2006; 200: 447-450
}

\author{
Objectives \\ To determine the range and frequency of diagnoses in specimens \\ submitted for histopathological examination by general dental \\ practitioners (GDPs). \\ Methods
}

A retrospective analysis was carried out of all cases submitted by GDPs for the period 1974-2003, using a FoxproTM Windows database. The data were collated into 10 diagnostic categories each comprising number of diagnoses, percentage of each diagnosis within a diagnostic category and each diagnosis as a percentage of total cases.

Results

GDPs submitted 6,666 cases out of a total of 53,474 for this period. While the total number of specimens increased four-fold over the $30-$ year period, specimens from GDPs increased from 7\% to 17\%. The range of diagnoses increased from 18 to 45 . Of the 617 GDPs who submitted material, 279 (45\%) submitted less than two specimens each in 30 years. Nine malignant neoplasms were diagnosed. Other significant pathology included 320 benign neoplasms as well as diagnoses ranging from mucosal lesions such as lichen planus to odontogenic cysts.

Conclusions

It is clear that GDPs have provided an increased number of biopsy specimens over the last three decades. This reflects an increasing demand by GDPs for a diagnostic oral histopathology service and their use of this service should be encouraged.

\section{IN BRIEF}

- Presents the range and relative frequency of histological specimens submitted by GDPs.

- Few GDPs regularly submit specimens despite the importance of histopathological confirmation of a clinical diagnosis.

- Small incisional and excisional biopsies are within the scopes of specialist practitioners and GDPs.

- The quality of specimens submitted by GDPs is similar to that of their hospital colleagues.

\section{COMMENT}

The last 30 years has been a period of considerable change in dental education, the general dental services and oral pathology services. Practitioners are sending more biopsy specimens, tackling a more diverse range of lesions and occasionally identifying lesions with important health implications for their patients. This is good news. Some have voiced the opinion that practitioners should not perform biopsy, on the basis that lesions with a high risk of malignancy are better sampled in hospital. However, many lesions seen and treated in general practice also merit biopsy. The principle that tissue worth excising is worth examining histologically is a good one.

At the start of the study period the concept of the dentist as oral physician was being strongly promoted, with a change in emphasis towards the dentist as oral diagnostician and guardian of oral, rather than dental, health. It is therefore perhaps surprising to see that despite a fourfold increase in submission of specimens from general practice, the typical GDP performs a biopsy very rarely. Data from Western populations ${ }^{1,2}$ suggest that oral lesions are common and it is intriguing to know whether, in the UK, they are being submitted elsewhere, being managed without the benefit of histological diagnosis or remaining undetected.

During the study period undergraduate teaching has evolved. There has been increasing emphasis on the processes of differential diagnosis and knowing when, and when not to, perform a biopsy rather than on learning the histological appearances of lesions. ${ }^{3}$ Unfortunately, reductions in undergraduate clinical experience mean that students may well qualify without having performed a biopsy, perhaps even without having observed the procedure. Given the important diagnoses reported in this paper, does this indicate a potential for harm

to the patient?

There has been debate about the financial disincentive to perform biopsy in general practice. The fee bears no relation to costs or the potential value of the examination. However, as the authors point out, departments of oral and maxillofacial pathology continue to provide a diagnostic service for practitioners on a charitable basis. There is certainly a need to resource this service appropriately, publicise it effectively and promote appropriate biopsy and diagnosis in the primary care setting.

E. W. Odell, Professor of Oral Pathology and Medicine, King's College London Dental Institute at Guy's Hospital

1. Axéll T. A prevalence study of oral mucosal lesions in an adult Swedish population. Odontologisk Revy 1976; 27: supplement 36.

2. Bouquot J E. Common oral lesions found during a mass screening examination. J Amer Dent Assoc 1986; 112: 50-57

3 Odell E W, Farthing P M, High A et al. British Society for Oral and Maxillofacial Pathology UK Minimum Curriculum in Oral Pathology. Eur J Dent Education 2004 8: $177-184$.

doi: $10.1038 /$ sj.bdj.4813465 